WORK IN PROGRESS

\title{
A visualization tool to support the decision making process of managers and caregivers from geriatric residences
}

\author{
Amanda Tapia Zazueta, Jessica Beltrán Márquez, \\ Valeria Soto Mendoza
}

Published: 21 September 2016

\begin{abstract}
Geriatric residences are companies dedicated to provide care to older adults. Usually, the number of residents surpasses the number of caregivers producing that the caregivers are not fully aware about the state of the residents. We propose the design of a visualization tool with the purpose of depict useful and relevant information to caregivers and managers to intuitively and rapidly interpret graphic indicators to know more about the state of the residents and improve the quality of service.
\end{abstract}

Keywords: Geriatric residences; visualization; behaviors.

\section{Introduction}

Older adults who suffer from a chronic disease or dementia might no longer be able to perform their daily activities by themselves and need assistance from a caregiver [1]. In some cases, their family members enroll older adults into geriatric residences, which are enabled places where several residents live while they receive attention from a qualified staff. Usually, the number of residents surpasses the number of caregivers, causing that one caregiver be in charge of several residents. In addition, the different shifts during the day provoke that one resident receive assistance from different caregivers. A common procedure followed in geriatric residences requires that the staff keep manual logs to register the activities performed by the residents [2]. The annotated information is useful to have awareness about the state, wellbeing and the complete record from each resident. Additionally, if a relative ask about the condition from the older adult, the manager or the staff can use the data annotated to answer to specific questions regarding the older adult. Furthermore, the annotations will serve to managers to make decisions related to the operational and strategic part of the business, for example by measuring how and how much the

Tapia Zazueta, A., Beltrán Márquez, J.

Sonora Institute of Technology

Antonio Caso S/N and Kino

Cd. Obregón Sonora, México

Email: amandatapiaz@hotmail.com, jessica.beltran@itson.edu.mx

Soto Mendoza, V.

Center for Scientific Research and Higher Education at Ensenada

Tijuana-Ensenada highway 3918

Email:vsoto@cicese.edu.mx caregivers interact with each resident and to know the specific needs from each resident. Unfortunately, caregivers are not usually aware of the full history of each individual resident due to their shifts, so they do not review previous annotations.

Recently, technological tools have been proposed in order to support the caregiving of older adults. For example, it has been proposed a system to support the capture of notes using electronic recording [3]. By using this system, each caregiver carries a smartphone where they can register the activities performed by each resident using a set of predefined activities. This information is sent to a server where the data registered from all caregivers is collected. This data can be used to generate knowledge relevant for managers, physicians and caregivers from geriatric residences.

An approach to interpret the collected annotations consist in designing algorithms to automatically detect patterns that can be used to detect behaviors. For example, it has been proposed an algorithm to detect if the residents are having normal or abnormal behavior [4]. However, this automatic recognition remains as an open problem.

An alternative approach consists in presenting the information to the staff through visualization tools. The aim of these tools is to depict important key indicators through graphical representations [5]. The information displayed must be useful to help to the staff in the decision making process. For example, by just a look at the indicators, a new caregiver easily, at the beginning of the shift, should be aware about the state of the residents that he/she is about to assist and understand how to approach to a specific condition of the resident. Another example consists in using the visualization tool to detect if the residents are having a normal or abnormal day. This later can be used to trigger an alert on the staff about a possible disease, depression and he/she can decide about medical intervention or informing to their relatives.

In this paper, we propose the design of a visualization tool to support companies dedicated to geriatric assistance. We propose to use the electronic records of caregivers' annotations to help to the managers and to the staff to answer questions about the wellbeing and status of the residents. We are particularly interested in the design of a graphical tool to visually detect normal or abnormal behaviors from residents. In the next section we show related work, in section 3 we state design considerations and in section 4 we present the closing remarks. 


\section{Related work}

Visualization tools have been adopted in the domain of business with the purpose of supporting the decision making process. This is achieved by converting raw data into knowledge through a proper display of the key indicators. For example, the company Celesquest have proposed dashboard tools to visualize earnings and sales that enables to executives to observe displays that provide an overview of the performance of a company, showing a set of related data [6].

Some visualization tools consider obtaining knowledge for health purposes like monitoring self well-being. For example, it has been proposed a visualization tool for displaying health related data in smart homes. The visualization includes physical, cognitive, social and spiritual information from an individual. This visualization tool was evaluated through focus groups with older adults in [7]. An important remark from this study is that health monitoring systems can collect a substantial amount of data that can be used along with visualization tools to provide a meaningful overview of long-term health progress. Another example is given by the ORCATECH life laboratory platform [8]. In this project, the data is captured through motion sensors installed in homes, that detect the location of the person, and the display is achieved using a two-dimensional spiral plot. The purpose of this visualization tool is to eyeball changes in routines or possible diseases. As a downside, it lacks on highly labeled semantic information like the activities performed from the person.

Community visualization dashboards have been proposed with the objective of serving as a tool to understand the indicators that affect the quality of the life of the residents based on their wealth. An example of the information depicted is showing statistics about adults with diabetes, by age, gender, ethnicity and the presence of cases of diabetes distributed by locations in maps of the community [9].

Up to our knowledge, there is not related work about visualization tools designed for geriatric residences that can be used to monitor the activities from the residents, to eyeball changes in their routines and to serve to managers to understand the process of the service offered by the company.

\section{Proposed solution}

In this paper, we propose a visualization tool to display relevant data for geriatric residences. Even though the potential of this tool can cover many aspects from the geriatric residence company, in this paper we focus on proposing a design for depicting the data collected from annotations of the activities from residents, that as mentioned earlier it is useful for caregivers and managers as well.

To illustrate a potential application from the proposed visualization tool, we show Figure 1 depicting the activities performed from an older adult living in a geriatric residence during 10 days. The information displayed corresponds to data collected in a real scenario. This data was captured in a sensing campaign conducted in various geriatric residences [4]. The collection was performed using electronic activity logs using smart phones during 6 months. The total data obtained consist in 19.182 activity logs from 15 residents with an average of 91 days annotated per resident and an average number of 8.63 activities per day. The recorded activities were: Anomaly, Check over. Toilet, Inside, Outside, Medication, Hygiene, Mood, Recreation, Social interactions, Feeding and Vitals.

The proposed design in Figure 1 emerged from a preliminary analysis of the collected data. We consulted to the researchers that conducted the sensing campaign. Their familiarization with the data helped us to organize the information and to identify how to display behavior patterns.

In Figure 1, the information from resident 5 is shown. The column of the left shows the colors of each activity and the 3D spiral graph in the right shows when the activities were annotated for the resident 5 during the last 10 days. Each circle corresponds to a specific day and the time over the day is distributed clockwise. The circle near to the center correspond to 10 days ago while the circle in the front corresponds to the current day. As we can see from the distribution of the annotated activities we can

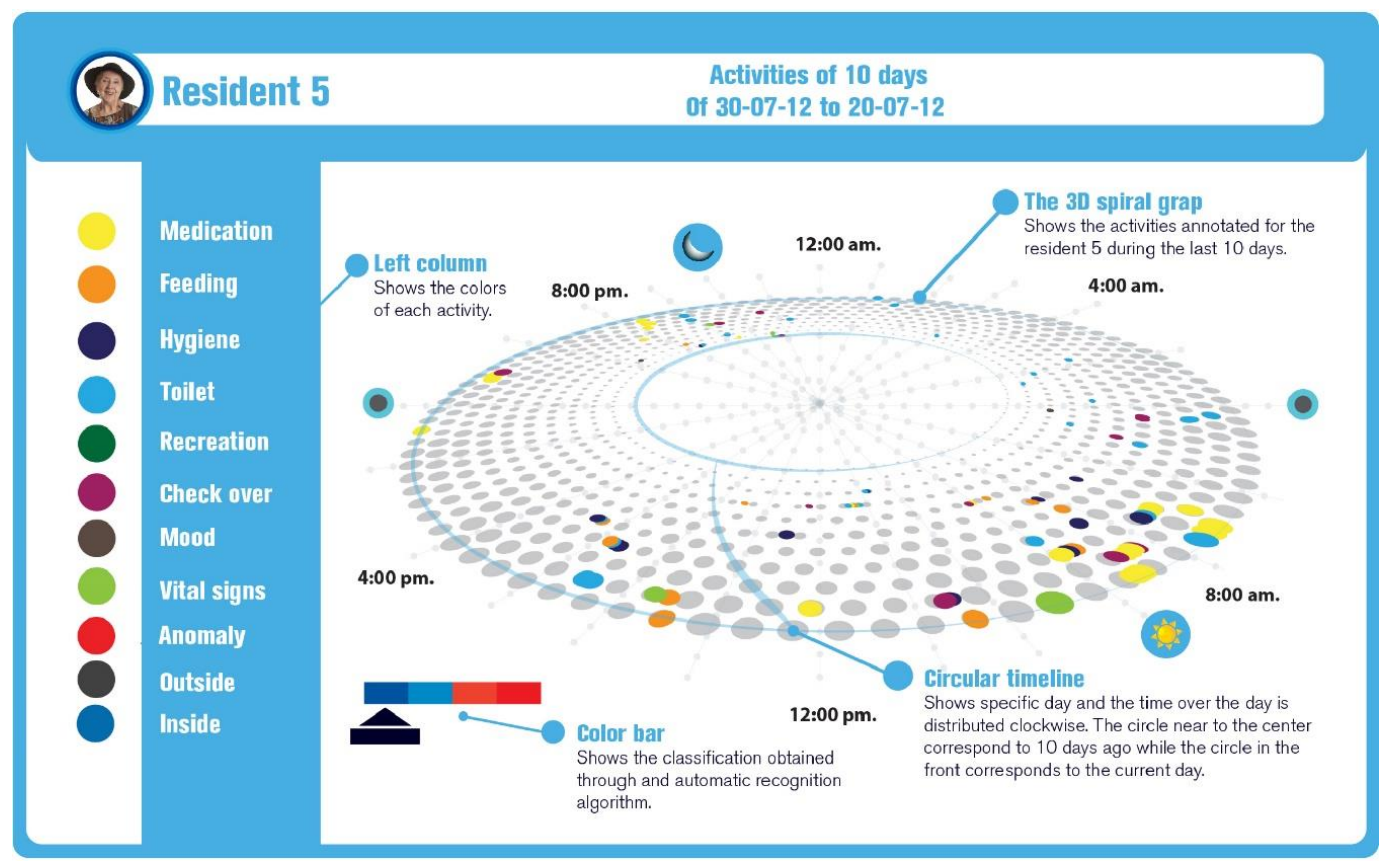

Figure 1. Example visualization tool with the activities of the resident 5 
eyeball that residents 5 usually takes medication at $8 \mathrm{am}$ and at $8 \mathrm{pm}$. However, we can see that one day he/she received medication at $12 \mathrm{pm}$ and at $7 \mathrm{pm}$ followed by vital checking at $1: 30 \mathrm{pm}$ and an unusual use of the toilet at $2 \mathrm{pm}$. This could be an indication of a possible disease. Nevertheless, the caregivers or the physician are the ones prepared to interpret properly what this information means. In this sense, the visualization tool permits the spotting and interpretation from persons related to the residents which enrich the decision making process comparing to the decision that an automatic recognition algorithm could take. Still, the color bar depicted at the bottom can show the classification obtained through and automatic recognition algorithm like the one proposed in [4].

A visualization tool as proposed could be used to determine the following:

- Spot routines or change in routines from residents.

- Trigger alert of possible disease.

- Determine normal or abnormal behaviors.

- Know who are the residents that each caregiver interacts with.

- Know who are the caregivers that each resident interacts with.

- Spot special needs from a resident (like the hours he/she is awake, when prefers to bath or eat, etc.).

The aim of our proposal is that later can be used as a business intelligent tool with the purpose to serve as interactive process to explore and analyze structured information to discover trends and patterns that will be used to derive conclusions [10]. In this paper we only propose depicting the data of activities from the residents but there are other aspects from the company that can be incorporated in the visualization tool. For example, the expenses and earnings in the provided services, to keep track of the information related to employees, about the medicines or food intake among others, which can be displayed depending on the user profile: caregivers, managers, family, etc.

\subsection{Future work}

The design of the visualization tool proposed is incipient. However, it just emerged from the analysis of the data set and talks with researchers. We are aware that methodologies used in Human Computer Interaction will improve the design after some rounds of iterative design sessions with the users. This is paramount to achieve a proper display of the activities' annotations and to have an intuitive interaction.

The proposed visualization tool will be used by the managers and the caregivers from geriatric residences. For that reason, is necessary to conduct interviews and focus group with them with the purpose of obtain more information about what they consider the key indicators to be depicted and what is considered a proper graphical representation. Some examples of the information that we can obtain through this interviewing process includes: if the 3D spiral graph is adequate, how many days are convenient to depict, the interaction followed to view the information from different residents, the colors and any other ideas that could emerge.

The following step consist in propose design solutions based on the ideas and specify requirements from the users. In Figure 1 we show the design obtained with the ideas from the researchers that conducted the sensing campaign but is necessary to know the needs from the users. Next, the proposed designs will be evaluated through usability testing. In the evaluation testing we can design scenarios of normal/abnormal days from residents and ask to the users to interact with the visualization tool in order to validate the interaction and the ability to properly display the patterns from the residents.

\section{Closing remarks}

The caregivers and managers from geriatric residences interact with different residents who produce a large amount of information through the performance of activities. This information can be useful to know the state of the residents or for decisions concerning to the company. Since sometimes is difficult to keep track of the state from all residents, a visualization tool is helpful to improve the quality of the provided service.

Methodologies from HCI will help to achieve a proper design and to investigate with the users what and how the information will be depicted. Additionally, the evaluation stage with final users will validate our proposal.

We plan to enhance the visualization tool by including more information respecting to the geriatric residences. For example, to analyze data regarding the money from the company. To keep track of the use of the supplies, about the development of employees, etcetera. For this purpose, we need to get more involved with the process followed in this type of company.

\section{References}

[1] Katz, S. 1983. Assessing self- maintenance: activities of daily living, mobility, and instrumental activities of daily living. Journal of the American Geriatrics Society, 31(12), 721-727.

[2] Soto-Mendoza, V., \& Garcia-Macias, J. A. (2014, December). Lessons learned from a long-running assistive system for geriatric care. InInternational Workshop on Ambient Assisted Living (pp. 83-90). Springer International Publishing.

[3] Soto-Mendoza, V., García-Macías, J. A., Chávez, E., Martínez-García, A. I., Favela, J., Serrano-Alvarado, P., \& Rojas, M. R. Z. 2015. Design of a Predictive Scheduling System to Improve Assisted Living Services for Elders.ACM Transactions on Intelligent Systems and Technology (TIST), 6(4), 53.

[4] Soto-Mendoza, V., Beltrán, J., Chávez, E., Hernández, J., \& García-Macías, J. A. 2015. Abnormal behavioral patterns detection from activity records of institutionalized older adults. In Human Behavior Understanding (pp. 119-131). Springer International Publishing.

[5] Elias, M. 2012. Enhancing User Interaction with Business Intelligence Dashboards (Doctoral dissertation, Ecole Centrale Paris).

[6] Few, Stephen. 2006. Information dashboard design: The effective visual communication of data, O' Reilly.

[7] Le, T., Reeder, B., Yoo, D., Aziz, R., Thompson, H. J., \& Demiris, G. 2015. An evaluation of wellness assessment visualizations for older adults. Telemedicine and e-Health, 21(1), 9-15.

[8] Life Lab 2016. Oregon Health \& Science University. Retrieved from http://www.ohsu.edu/xd/research/centersinstitutes/orcatech/tech/life-lab.cfm

[9] Placer, B. 2016. Be Well Placer [online] Placerdashboard.org. Available at: http://www.placerdashboard.org/index.php?module=Tiles\&c ontroller $=$ index $\&$ action $=$ display $\& i d=64842804736729988$. [Accessed 6 Jul. 2016].

[10] Cano, J. L. 2007. Business intelligence: competir con información. Banesto, Fundación Cultural. 